\title{
Betrachtungen über Skoliose
}

\section{anknüpfend an eine Besprechung der Lorenz'schen Monographie.}

Von Dr. H. Nebel in Hamburg.

(Fortsetzung aus No. 28.)

Die nachstehende Tabelle (s. p. 648 und 649) ist mit peinlichster Gewissenhaftigkeit ans mehreren Hundert Messungsbildern von 82 Patienten zusammengestellt.

Von den Gemessenen waren 11 männlichen Geschlechts, (davon 6 zwischen 10 und 15 Jahre, 3 bis 18,2 über 20 Jahre [ 21 und 25 ] alt),

71 weiblichen Geschlechts,

davon 15 zwischen 5 und 10 Jahren

$36 \quad 10,15 \%$ (wovon 22 im 14. oder 15)

$17 " \quad 16 \quad " 20 \quad$,

2 über $\quad 16 \quad 20$ " (nämlich 25 und 28 Jahre alt)

Die Angaben über Körpergrösse und Constitution zeigen, dass es sich, abgesehen von den rhachitisch Beanlagten, in der Mehrzahl der Fälle um zarte, schlanke, oft sehr rasch gewachsene Individuen handelte.

Der Schulterstand war 18 mal rechts und links der gleiche

$$
4 \text { mal ",". "abwechselnd; }
$$
mit nur 3 Ausnahmen handelte es sich dabei um Fälle mit compensatorischen Gegenkrümmungen.

$$
\begin{aligned}
& 23 \text { mal stand die linke Schulter tiefer } \\
& 30 \text { mal fo " rechte " } \\
& 7 \text { mal fehlen Notizen über diesen Punkt. }
\end{aligned}
$$

Der Abstand der Schulterblattspitzen von der frontalen Medianebene (nach hinten) ist graphisch dargestellt, so zwar, dass die Linie von oben (am Kopfe der Zahl) wach unten rechts (am Fusse der Zahl) verläuft, wenn die rechte Schulterblattspitze mehr nach hinten vorragt und umgekehrt. Die zu beiden Enden der Linie notirten Zahlen geben die Entfernung der Schulterblattspitzen von der sagittalen Medianebene an; die Ziffer der höher hinauf gerückten Schulterblattspitze ist unterstrichen. Zweimal war eine Linksabweichung der Wirbelsäule so stark, dass auch die rechte Schulterblattspitze noch links von der Mittellinie zu stehen kam, es ist dies durch ein der Ziffer vorgesetztes Minuszeichen angedeutet. Wo der Stand der Schulterblattspitzen bei wiederholt vorgenommenen Messungen bald rechts, bald links höher war, steht ,wechselnd" auf der horizontal, - ebenso wie bei Gleichstand der Schulterblattspitzen gehaltenen Linie. Fast in der Hälfte aller Fälle istes die rechte Schulterblattspitze, welche mehr nach hinten absteht; dahei liegt aber keineswegs immer oder auch nur in der Mehrzahl der Fälle rechtsconvexe Dorsalskoliose zu Grunde.

Die Abweichung der Wirbelsäule von der Mittellinie war nur nach einer Seite hin gerichtet in 33 Fällen;

in 36 Fällen bestand einfache compensatorische Ausbiegung

, 12

12 Falle " $\quad$ dreifache ,

Meist betrafen "die einfachen Ausbiegungen noch nicht lange bestehende, bewegliche, in der Entstehung begriffene Skoliosen, es waren aber auch mehrmals alte, längst fixirte Deviationen (No. 12. 14. 59.) allerhöchsten Grades. Die Haupt- resp. ausschlaggebende Verkrümmung ist vorangestellt; in den wenigen Ausnahmefällen, wo ihr nicht die höhere Ziffer (die Grösse der Abweichung im Scheitel des Bogens angebend, entspricht) liess die der voranstehenden Deviation entsprechende Torsionserhebung am Rücken keinen Zweifel darüber aufkommen, dass hier die primäre Biegung zu suchen sei.

$$
\text { (Fortsetzung folgt auf p. 650.) }
$$




\begin{tabular}{|c|c|c|c|c|c|c|c|c|c|c|c|c|}
\hline 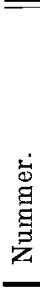 & 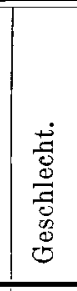 & 宫 & 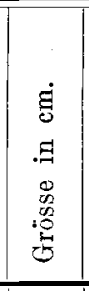 & $\begin{array}{l}\text { tiefer } \\
\text { stehende } \\
\text { Schulter. }\end{array}$ & $\mid \begin{array}{c}\text { Abstand der } \\
\text { Schulter- } \\
\text { blattspit- } \\
\text { zen nach } \\
\text { rückwarts } \\
\text { (graphisch) } \\
\text { nnd vonder } \\
\text { Mittellinie } \\
\text { (in cm) } \\
\text { (die hoherste- } \\
\text { hende unter- } \\
\text { l. strichen) r. }\end{array}$ & $\begin{array}{l}\text { Form und Grösse } \\
\text { der } \\
\text { seitl. Verbiegung. }\end{array}$ & $\begin{array}{l}\text { antero-posteriore } \\
\text { Rückenlinie. }\end{array}$ & sup. & anter. & 范 & $\begin{array}{l}\text { Becken- } \\
\text { Assyme- } \\
\text { trie. }\end{array}$ & Constitution und Bemerkungen. \\
\hline 1. & fem & $?$ & 113 & linke & & $\begin{array}{l}\text { dors. d. }(0,5)- \\
\text { lumb. s. }(0.5)\end{array}$ & Starke Dorsalkyphose. & 63,5 & 62,5 & r. -1 & & Mager, anaemisch. \\
\hline 2. & fem. & 15 & 150 & linke & $6-8$ & $\left|\begin{array}{l}1 \mathrm{u} \mathrm{m} \text { b. s. }(1,8)- \\
\text { dors. d. }(1,3 \text { cerv. } \sin .)\end{array}\right|$ & $\begin{array}{l}\text { Absolut flach zwischen den } \\
\text { Schulterblättern, sehr starke } \\
\text { Lendenlordose. }\end{array}$ & 85 & 86 & $1 .-1$ & B.-Ass. & Klein, anaemisch, rhachit. (?) \\
\hline 3. & fem. & 12 & 150 & $=$ & & tot. $\sin .1,2$ & Flacher Rücken. & 83 & 83 & & & Blass, Muskelschwäche. \\
\hline 4. & fem. & 13 & 148,5 & rechte & $\frac{8, \overline{5}}{-}-0,5$ & $\begin{array}{c}\text { dors.sin. }(3)-1 . \text { d. } \\
(0,5)\end{array}$ & Starke Lendenlordose. & 88 & 88,5 & $1 .-0,5$ & & Schlank, rasch gewachsen. \\
\hline 5 . & $\mathrm{m}$. & 25 & 160 & linke & $1,5-14$ & $\begin{array}{c}\text { dors. dextr. }(3,5) \\
-1 . \text { s. }(0,3)\end{array}$ & $\begin{array}{l}\text { Starker rs. Rippenbuckel } \\
\text { und starke Lendenlordose. }\end{array}$ & 96,5 & 95,5 & r. -1 & B.-Ass. & Rhachit. (?) \\
\hline 6. & fem. & 17 & 155 & linke & & $1 \mathrm{umbal}$. $\sin .(2,0)$ & Starke Liendenlordose. & 91,5 & 92,7 & $1 .-1,2$ & B.-Ass. & Schlank, blass. \\
\hline 8. & fem. & 25 & 161 & $=$ & 8,5 & $\begin{array}{c}1 \mathrm{u} \mathrm{m} \text { b. } \mathrm{d} \cdot(2,5)-\text { dors. } \\
\sin .(1,2)\end{array}$ & etwas flacher Rücken. & 91,5 & 92,6 & $1 .-1,1$ & B.-Ass. & $\begin{array}{l}\text { Lehrerin. Beginn des Leidens im } 13 . \\
\text { Lebensjahr und stetige Zunahme } \\
\text { trotz Corsetbehandlung bis jetzt. }\end{array}$ \\
\hline 9 . & fem. & 14 & 147 & wechselnd & $1-10$ & $\begin{array}{c}\text { dors. dextr. } \\
\text { (cervic. } \sin .)\end{array}$ & $\begin{array}{l}\text { rs. Rippenbuckel u. st. } \\
\text { Lendenlordose. }\end{array}$ & 89,7 & 87,5 & $\mathrm{r} .-2,5$ & B.-Ass. & Rhachit. blass. \\
\hline 10. & fem. & 16 & 152 & linke & $\underline{3}-10$ & $\begin{array}{l}\text { dors. d. }(5)-\text { lumb. } \\
\text { s. }(0,6) \text { (cervic. sin,) }\end{array}$ & $\begin{array}{l}\text { rs. Ripp e nbucke } 1 \text { u. st. } \\
\text { Lendenlordose. }\end{array}$ & 88,2 & 89 & $1 .-0,8$ & & Rhachit. \\
\hline 11. & fem. & $i 2$ & 149 & rechte & & $\begin{array}{c}\text { do rs. d. }(1,6)-1 . \\
\text { sin. }(0,4)\end{array}$ & Flacher Rücken. & 85 & 85 & & B.-Ass. & \\
\hline 12. & fem. & 17 & 143,5 & linke & 1 & dors. d. (6) & $\begin{array}{l}\text { rs. Rip p enbuck el } \\
\text { st. Lendenlordose. }\end{array}$ & 83 & 81,5 & $r .-1,5$ & B.-Ass. & Rhachit. (?), klein, blass. \\
\hline 13. & fem. & 14 & $15 \pm, 5$ & $=$ & $4-7,5$ & $\left|\begin{array}{l}\text { dors. d. }(3)-1 . s . \\
(0,5) \text { d.-cerv. s. }(0,5)\end{array}\right|$ & $\begin{array}{l}\text { rs. Rippenbuckel } \\
\text { st. Lendenlordose. }\end{array}$ & 90,4 & 90,4 & & & Sehr anaemisch. \\
\hline 14. & $\mathrm{~m}$. & 18 & 149 & linke & $4+-11$ & dors. d. (8) & $\begin{array}{c}\text { coloss. r. s. R ipp en bu cke } 1 \\
\text { und Dorsalkyphose. }\end{array}$ & 100 & 98 & r. -2 & & Rhachit., schwach. \\
\hline 15. & fem. & 18 & 157 & linke & & dors. d. $(3,7)$ & Starke Lendenlordose. & 93,9 & 94,2 & $1,-0,3$ & B.-Ass. & Schwach, anaemisch. \\
\hline 16. & fem. & 17 & 154 & rechte & $\underline{9,5}-\overline{3}, 5$ & dors. $\sin .(5)$ & $\begin{array}{l}\text { coloss. ls. Rippen- } \\
\text { buckel u. hohe Dorsalky- } \\
\text { phose. }\end{array}$ & 97,7 & 97,5 & & B.-Ass. & Rhachit. 一 Vitium cordis. \\
\hline 17. & fem. & 17 & 159 & $=$ & & tot. $\sin .(1,3)$ & & 93 & 92 & r. -1 & & Gross, zart, anaemisch. \\
\hline 19. & fem. & 14 & 158 & linke & & total. sin. $(0,5)$ & $\begin{array}{l}\text { Verstärkte Dorsal- resp. } \\
\text { Dorso-cervic.-Kyphose. }\end{array}$ & 93 & 93 & & & Sehr mager, blass. \\
\hline 20. & fem. & 17 & 166,5 & rechte & $5,5-8,5$ & total. d. $(1,5)$ & St. Dorsalkyph. (r. Rücken). & 96,3 & 95,5 & $r .-0,8$ & B.-Ass. & Anaemisch, scrophulös. \\
\hline 21 . & fem. & 15 & 164 & rechte & 4 & total. d. (1) & Hoher runder Rücken. & 96 & 96 & & & Sehr rasch gewachsen. \\
\hline 22. & m. & 11 & 137 & wechselnd & 3,5 & total. sin. $(1,2)$ & Hoher runder Rücken. & 76,5 & 76,5 & & B.-Ass. & Rhachit. (?) \\
\hline 23. & fem. & 13 & 150 & linke & $\begin{array}{l}-8 \\
7,5\end{array}$ & $\begin{array}{l}\text { dors. dextr. }(1)- \\
\text { l. s. }(0,3)-\text { cervic. } \\
\text { dors. s. }(0,3) \\
\text { dors. d. }(1,0)-1 . \mathrm{s} . \\
(0,2) \text { cerv.-d. s. }(0,2)\end{array}$ & $\begin{array}{c}\text { Rippenhöcker rs. starke } \\
\text { Dorsalkyphose u. tiefe } \\
\text { Lendenlordose. }\end{array}$ & 92 & 86,5 & r.- -1.5 & & Rhachit. (?) anaem., muskelschwach. \\
\hline 25. & fem. & 13 & 131,5 & $=$ & 4 wechs. 7 & $\begin{array}{l}\text { d ors. d. }(1,2)-1 . \mathrm{s} \\
(0,2) \text { cervic.-s. }(0,2)\end{array}$ & & 74,3 & 74,2 & & & Rhachit. zart. \\
\hline 26. & fem. & 12 & 137,5 & wechselnd & 4. wechs. 6 & $\begin{array}{l}\operatorname{lumb} \text {-dors. sin. } \\
(1,3)-\text { dors. d. }(0,4)\end{array}$ & flacher, oben runder Rücken. & 81 & 81,5 & $1 .-0,5$ & B.-Ass. & Zart, blass. \\
\hline 27. & fem. & 16 & 162,5 & rechte & $\underline{6}-8$ & $\begin{array}{l}\text { dors. } \sin .(6,5)-1 . \\
\text { d. }(0,5)\end{array}$ & $\begin{array}{c}\text { coloss. Ripp en buckel lin- } \\
\text { kerseits u. Dorsalkyphose, } \\
\text { tiefe Lendenlordose. } \\
\text { Hoher runder Rücken. }\end{array}$ & 100,5 & 99,5 & r. $-0,5$ & B.-Ass.? & $\begin{array}{l}\text { Beginn des Leidens im 11. Jahr nach } \\
\text { Scharlach, stete Zunahme trotz } \\
\text { unausgesetzter Behandlung (Hăngen } \\
\text { am Kopfe und Gyps, Filzcorsets). } \\
\text { Sehr rasch gewachsen. }\end{array}$ \\
\hline 29. & fem. & & 116,5 & rechte & & total. sin. (3) & $\begin{array}{l}\text { Ls. Rippenbuckel und } \\
\text { st. Dorsalkyphose. }\end{array}$ & 62,2 & 62,2 & & & Rhachit. klein. \\
\hline 30. & fem. & 91 & 120 & linke & & $\begin{array}{c}\text { dors dextr. }(3,3)- \\
\text { lumb. sin. (1) }\end{array}$ & $\begin{array}{l}\text { Rs. Rippenbuckel u. } \\
\text { sehr starke Dorsalkyphose. }\end{array}$ & 68 & 67,5 & r. $-0,5$ & B.-Ass. & Rhachit. anaemisch, zart. \\
\hline 31. & fem. & 71 & 114.5 & linke & $2,5-10$ & $\begin{array}{c}\text { dors. d. }(2)-\text { lumb. } \\
\text { sin. }(0,5)\end{array}$ & Starke Dorsalkyphose. & 63,5 & 62,3 & r. $-1,1$ & B.-Ass. & Rhachit. \\
\hline 32. & fem. & 141 & 155 & linke & & $\begin{array}{c}\text { dors. d. }(1,5)-1 . s . \\
(0,7)\end{array}$ & Hoher runder Rücken. & 90,5 & 90,5 & & B.-Ass. & Gross, blass. \\
\hline 33. & fem. & 81 & 122,5 & $=$ & & $\begin{array}{l}\text { dors. d. }(0,5)-\text { d.- } \\
\quad \text { cerv. } \sin .(0,5)\end{array}$ & Hoher runder Rücken. & 68 & 68 & & & Mager, zart. \\
\hline 34. & fen. & 201 & 165,5 & $=$ & & $\begin{array}{l}\text { dors. d. }(2,5)-1 . \mathrm{s} \\
(0,8)-\text { d.-c. s. }(0,3)\end{array}$ & Dorso-cervic.-Kyphose. & 96,5 & 97,5 & $1 .-1,1$ & & Sehr zart, anaemisch. \\
\hline 35. & fem. & 101 & 135,5 & $=$ & 4,5 weechs. 7 & total. dextr. $(0,6)$ & Hoher runder Rücken. & 79 & 78,5 & r. $-0,5$ & & \\
\hline 36. & fem. & 141 & 155 & rechte & & $\begin{array}{c}\text { dorso-cer v.d. }(2,0) \\
- \text { lumb. sin. }(1,0)\end{array}$ & Hoher runder Rücken. & 92 & 92,5 & $1 .-0,5$ & & Rhachit. (?) \\
\hline 37. & m. & 111 & 139 & rechte & & total. dextr. $(1,0)$ & Hoher runder Rücken. & 80,8 & 81 & & & Sehr anaemisch. \\
\hline 38. & fem. & 141 & 162 & rechte & $6, \overline{5}-7$ & $\begin{array}{l}\text { lumb. sin. }(0,8)- \\
\text { dorso-cerv. d. (1) }\end{array}$ & Hoher runder Rücken. & 94 & 94,8 & $1 .-0,8$ & & Sehr gross und schlank. \\
\hline 39. & fem. & 151 & 158 & rechte & 5,5 & $\begin{array}{c}1 \mathrm{um} \text { b. } \sin .(0,7)- \\
\text { dors. d. }(0,5)\end{array}$ & Hoher runder Rücken. & 90,7 & 90 & r. $-0,7$ & & \\
\hline
\end{tabular}




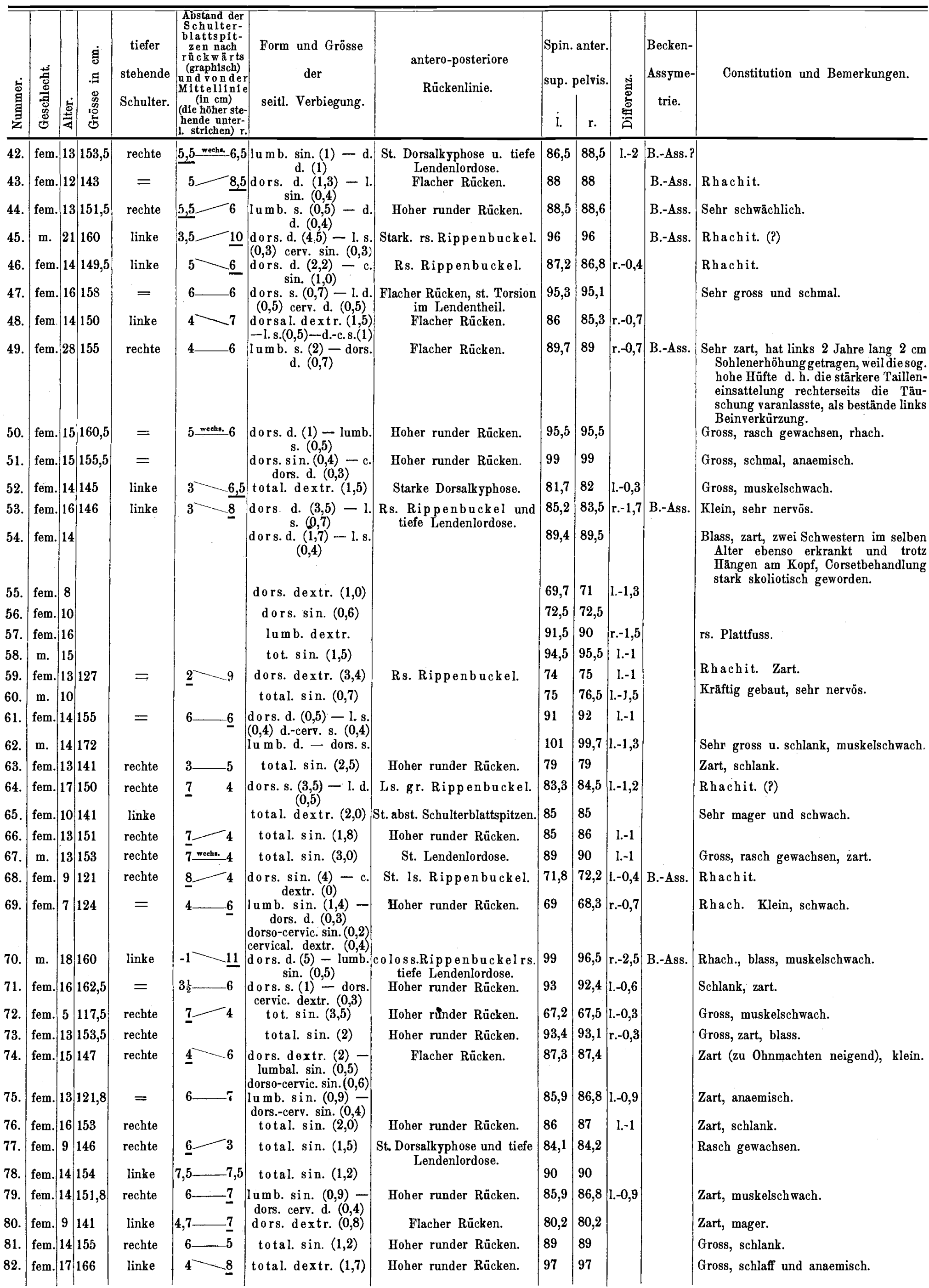


Ein deutlicher, mitunter colossaler Rippenbuckel bestand $18 \mathrm{mal}$, rechts $13 \mathrm{mal}$, links $5 \mathrm{mal}$; in der Mehrzahl aller Fälle verstärkte, Buckelhaltung (,hoher Rücken") bedingende Dorsalkyphose und oft verstärkte Lendenlordose.

Die rechte Spina pelvis stand $23 \mathrm{mal}$ tiefer (unter $0,5 \mathrm{~cm}$ 2 mal; unter $1,0 \mathrm{~cm} 9$ mal; zwischen 1 und $2 \mathrm{~cm} 9 \mathrm{mal} ; 2$ bis $3 \mathrm{~cm} 3 \mathrm{mal}$ ), die linke Spina pelvis stand $29 \mathrm{mal}$ tiefer (unter $0.5 \mathrm{~cm} 5 \mathrm{mal}$; unter $1,0 \mathrm{~cm} 7 \mathrm{mal}$; zwischen 1 und $2 \mathrm{~cm}$ $15 \mathrm{mal}$; 2 bis $3 \mathrm{ctm} 1 \mathrm{mal}$ und einmal $3 \mathrm{~cm}$ ), während sie $30 \mathrm{mal}$ gleich stehend befunden wurden.

Beckenasymmetrie bestand $27 \mathrm{mal}$, (worunter 2 fragliche Fälle, weil nur einmal gemessen); davon betreffen 9 Patienten mit Skoliosis lumbalis sin. oder dextra, 13 rhachitische Individuen. Ich habe schliesslich anch die Constitution angegeben, um darauf aufmerksam zu machen, dass es sich in erster Linie bei bestehender Skoliose um ein constitutionelles Leiden handeln dürfte, welches mit Vorliebe junge Mädchen dicht vor oder hinter der Pubertät befällt und besonders häufig schnell aufgeschossene, magere, anaemische Individuen gefährdet, welchen muskelstärkende Bewegungen (in Ausnahmefällen auch „Massage“ als theilweiser Eirsatz oder als schonendere Einleitung der Bewegungscur) gewiss in erster Linie zu empfehlen sind, da sie eines der wirksamsten Mittel darstellen, um die Gesammternährung und die vitale Energie zu verbessern.

Rhachitis konnte ich, bei aller Sorgfalt und Vorsicht bezüglich der Fragestellung, nur in 13 Fällen anamnestisch feststellen; in manchen Fällen aber, wo die Mutter fehlte, war über die Zahnung, Laufenlernen etc. des Kindes nichts zu eruiren. In weiteren 8 Fällen glaubte ich auf Grund der Schädelformation, Zahnbildung und dergleichen Rhachitis annehmen zu können.

Prognostisch hält Lorenz die primäre Lendenskolioses für die günstigste, zu deren Heilung ihm oft einige Monate ausreichen, während man bei primärer Dorsalskoliose nicht selten froh sein müsse, nur weiteres Fortschreiten der Verkrïmmung aufzuhalten. $O b$ es wirklich, wie Lorenz annimmt, unmöglich ist, die convexseitigen Rippenwinkel noch etwas abzuflachen, möchte ich nach dem, was ich von heilgymnastischer Behandlung erfahren habe, dahingestellt sein lassen; allzuviel wird man freilich nicht erwarten dürfen. Ich glanbe, dass von der seitlichen Suspension, wie sie L o renz empfiehlt, und von Beely's Rückenschwinge in dieser Hinsicht manches Gute zu erwarten ist, natürlich nur bei solchen Individuen, die noch im Wachsthume begriffen sind.

Die rhachitischen Skoliosen geben nach Lorenz eine sehr schlechte Prognose; bei Verwendung des Stehbettes ist dieselbe jedoch, wie Dr. Nönchen ${ }^{1}$ ) gezeigt hat, bei kleinen Kindern sogar eine recht gute.

Indem er p. 156 zur Therapie der Skoliose kommt, lässt Lorenz sich zunächst über den Werth der schwedischen Heilgymnastik in sehr absprechender Weise aus. Ich habe meinen Standpunkt zu dieser Frage schon im Vorhergehenden angedeutet und vermuthe, dass auch Lorenz, trotz seiner rein theoretischen Bedenken gegen die Inangriffnahme der Skoliose mittelst Bewegungen, anders urtheilen würde, wenn er Gelegenheit gehabt hätte, sich von den Vorzügen und Leistungen rationell gegebener schwedischer Heilgymnastik zu überzeugen. Wahrscheinlich aber hat er seine Informationen über das Verfahren hauptsächlich, wie die meisten Aerzte, aus jenen Quellen geschöpft, welche die Sache bei wissenschaftlich denkenden Leuten geradezu in üblen Geruch bringen mussten, infolge offenkundiger Kritiklosigkeit und Uebertreibungen, wie sie von Industriellen, welche sich die Ausbeutung des von den Aerzten mit Unrecht verschmähten Verfahrens angelegen sein liessen, nicht anders erwartet werden konnten.

Ich habe mir Mühe gegeben, über Werth oder Unwerth jenes zuviel gepriesenen, wie geschmähten Verfahrens Klarheit zu gewinnen, indem ich die schwedische Gymnastik an der Quelle aufsuchte. Ich brauchte einige Zeit, ehe ich, von manchem Vorurtheil befreit, den guten Kern einer mit vielen Schlacken behafteten Sache, herausfinden wollte, umsomehr als ich sehen musste, dass auch in Stockholm der Mehrzahl nach Leute ohne die nöthigen Kenntnisse, welche also sich und Anderen keine Rechenschaft von ihrem Wirken abzulegen im Stande sind, ganz wie bei uns ihren Zauber mit der Heilgymnastik trieben. Ich durfte aber während eines mehrmonatlichen Aufenhaltes, im Verkehr mit einer Anzahl ebenso tüchtiger wie liebenswürdiger Aerzte, - beim Besuche der verschiedenen, unter ärztlicher Leitung stehenden Anstalten (von Dr. Zander's und Dr. Levertin's medico-mechan. Institut, Professor Jaederholm's orthopäd. Institut, der heilgymnast. Abtheilung des gymnast. CentralInstituts unter Professor Hartelius und Dr. M urrey) meine Zweifel an der Wirksamkeit der mechanischen Behandlung verlieren, weil ich Manches hören und sehen konnte, wozu mir bislang die Gelegenheit gefehlt hatte. Ich überzeugte mich, wie sehr vor Allen
Dr. Zander bemüht ist, den wissenschaftlichen Standpunkt festzuhalten und durch unermüdliches Forschen die Begründung und Erklärungen für einen vorzugsweise empirischen Heilzweig zu gebeu. Die Urtheile von Duval, Bührung, Behrend, Adams, welche Lorenz anführt, weil sie niemals Resultate von heilgymnastischer Behandlung gesehen hätten, scheinen mir daher weniger gegen den Werth jener Behandlung, als gegen die Objectivität jener Autoren zu sprechen; jedenfalls dürften sie nicht Beweiskraft genug besitzen, um die gegentheiligen Angaben von ebensovielen angesehenen schwedischen und deutschen Aerzten Lïgen zu strafen. ${ }^{1}$ )

(Fortsetzung folgt.)

1) Das Stehbett. Deutsche medicinische Wochenschrift 1886, No. 18. 